\title{
Clinical profile of oral pemphigus vulgaris in Thai patients
}

\author{
RUCHADAPORN KAOMONGKOLGIT ${ }^{1, A-G}$, WILAIRAT SARIDEECHAIGUL 2-4, B, D, \\ ORCID ID: 0000-0003-2055-6412 \\ PORAMAPORN KLANRIT2, 3, в, D, PENSRI PHOTHIPAKDEE2, 3, в, D \\ ${ }^{1}$ Department of Oral Diagnosis, Faculty of Dentistry, Naresuan University, Phitsanulok, Thailand \\ ${ }^{2}$ Department of Oral Biomedical Science, Faculty of Dentistry, Khon Kaen University, Khon Kaen, Thailand \\ ${ }^{3}$ Research Group of Chronic Inflammatory Oral Diseases and Systemic Diseases Associated with Oral Health, \\ Khon Kaen University, Khon Kaen, Thailand \\ ${ }^{4}$ Neuroscience Research and Development Group, Khon Kaen University, Khon Kaen, Thailand
}

A - Study Design, B - Data Collection, C - Statistical Analysis, D - Data Interpretation, E - Manuscript Preparation, F - Literature Search, $\mathbf{G}$ - Funds Collection

Summary Background. Pemphigus vulgaris (PV) is a rare, life-threatening autoimmune disease and varies in its clinical profile and epidemiology in different regions of the world.

Objectives. This study aimed to evaluate the demographic and clinical characteristics of oral PV patients in the lower north and northeast of Thailand.

Material and methods. A retrospective study of PV patients who visited the Oral Medicine Clinic, Dental Hospital, Naresuan University and Khon Kaen University during the 2010-2020 period.

Results. 21 oral PV patients were included. Among these, $16(76.2 \%)$ were female, and $5(23.8 \%)$ were male, with a female to male ratio of 3.2:1. The mean age of the patients was $47.7 \pm 14.2$ (range 25 to 74 ) years. 8 patients (38.1\%) had a history of systemic diseases, and hypertension was predominant. The mean duration of the disease was $6 \pm 3.42$ (range 1 to 12 ) months. The most common complaint at presentation was oral ulceration with a burning sensation. The most affected sites were the buccal mucosa and gingiva (80.9\%). Extraoral involvement was found in 14 patients $(66.7 \%)$. Patients who had oral lesions before skin lesions found the mean duration was $4.63 \pm 5.13$ (range 1 to 16 ) months. Most of the patients (61.9\%) were prescribed a combination of topical and systemic steroids. Conclusions. The present study illustrates the demographics and clinical characteristics of oral PV in Thai patients. Early diagnosis and timely treatment are critical for the patients. Since oral mucosa is often the first site of PV involvement, healthcare professionals must recognize the disease's clinical characteristics and manage PV patients appropriately.

Key words: pemphigus vulgaris, demography, retrospective studies, Thailand.

Kaomongkolgit R, Sarideechaigul W, Klanrit P, Phothipakdee P. Clinical profile of oral pemphigus vulgaris in Thai patients. Fam Med Prim Care Rev 2021; 23(4): 442-446, doi: https://doi.org/10.5114/fmpcr.2021.110357.

\section{Background}

Pemphigus is a rare and chronic vesiculobullous autoimmune disease characterized by intraepithelial bullae and erosion of the skin and mucosa. Pemphigus vulgaris (PV) is the most frequent and most severe form of pemphigus [1]. It is lifethreatening if untreated, occurring globally, typically between the fourth to sixth decades of life [1]. The incidences of PV vary between 0.1-0.5 cases per 100,000 population per year [2]. Previous reports showed that PV occurs in males to females, ranging from $1: 1$ to $1: 5[3,4]$.

The oral mucosa is the first site of involvement in $50-70 \%$ of cases and occurs in $90 \%$ of patients during the disease $[1,3]$. Any part of the oral cavity can be affected, and the most common locations are friction zones such as the buccal mucosa, gingiva, tongue, palate and lower lip [5]. It usually begins with blisters and painful erosions on the oral mucosa, followed by other mucous membranes and flaccid blisters on the skin [1, 2]. The lesions are multiple and present in different sizes and irregular shapes. The gingival lesion presents as desquamative gingivitis. The lesions may involve the vermilion border of the lips, forming hemorrhagic crusting $[1,5]$. Oral lesions lead to problematic eating, impairing one's general and nutritional status. Oral involvement may persist for several months before progressing to the skin or other mucous membranes $[3,6]$. Oral lesions may also be the only manifestation of the disease [1].
Early diagnosis and timely treatment are demanding for PV patients. Therefore, education to improve healthcare professionals' awareness and knowledge of the clinical oral presentation of PV is necessary. Pemphigus vulgaris is a worldwide disease and varies in its clinical profile and epidemiology in different regions of the world. It is rare, and little epidemiological data is available.

\section{Objectives}

This study aimed to evaluate the demographic and clinical characteristics of oral PV patients in the lower north and northeast of Thailand.

\section{Material and methods}

The present study was approved by the Naresuan University Institutional Review Board (NU-IRB-COA No. 402/2020) and the Khon Kaen University Ethics Committee in Human Research (HE632269). The retrospective study included 21 PV patients who visited the Oral Medicine Clinic, Dental Hospital, Naresuan University and Khon Kaen University during the 2010-2020 period. The diagnosis of patients with PV was clinically and histopathologically and/or direct immunofluorescent confirmed. 
In addition, patient information regarding age, gender, medical conditions and current medications, chief symptoms, duration of the diseases, distribution of oral and extraoral lesions, diagnostic methods, treatments, hospitalizations and oral habits regarding tobacco and alcohol consumption were evaluated. The data was analyzed to basic variation statistical values using SPSS (version 17.0; SPSS Inc., Chicago, IL, USA).

\section{Results}

Table 1 shows the clinical profile of oral PV patients. 21 PV patients were included. Among these, 16 (76.2\%) were female, and $5(23.8 \%)$ were male, with a female to male ratio of 3.2:1. The mean age of the patients was $47.7 \pm 14.2$ (range 25 to 74) years old. 8 patients (38.1\%) had a history of systemic diseases. Among these, the most common systemic diseases, in descending order (number, \%), were hypertension (4, 19\%), diabetes mellitus (2, 9.5\%), gastritis and peptic ulcer (2, 9.5\%), dyslipidemia $(1,4.8 \%)$, ischemic heart disease $(1,4.8 \%)$ and hepatitis $B$ (1, 4.8\%). Most patients were non-smokers and non-drinkers (20, $95.2 \%)$, with only one male patient being a smoker and drinker.

The most common complaint at presentation was oral ulceration with a burning sensation. The mean duration of this symptom was $6 \pm 3.42$ (range 1 to 12 ) months. The most affected sites were the buccal mucosa $(81 \%)$ and gingiva (81\%), followed by lip and labial mucosa (47.6\%), palatal mucosa (33.3\%), tongue (33.3\%) and floor of mouth (19.1\%) (Figure 1). Nikolsky's sign on gingival mucosa was positive in 19 patients (90.5\%). Extraoral involvement was found in 14 patients (66.7\%) (Figure 2). Patients who had oral lesions before skin lesions found the mean duration was $4.63 \pm 5.13$ (range 1 to 16 ) months. The diagnostic methods were biopsy for histopathological examination with/ without direct immunofluorescence in all patients, and direct immunofluorescence was conducted in $52.4 \%$ of patients.

\begin{tabular}{|c|c|c|c|c|}
\hline \multicolumn{2}{|c|}{ Demographics and characteristics } & \multirow{2}{*}{$\begin{array}{l}\text { Female (\%) } \\
16(76.2) \\
3(14.3) \\
\end{array}$} & \multirow{2}{*}{$\begin{array}{l}\text { Male (\%) } \\
5(23.8) \\
0(0.0)\end{array}$} & \multirow{2}{*}{$\begin{array}{l}\text { Total (\%) } \\
21(100) \\
3(14.3) \\
\end{array}$} \\
\hline Age (years) & $20-30$ & & & \\
\hline & $31-40$ & $3(14.3)$ & $0(0.0)$ & $3(14.3)$ \\
\hline & $41-50$ & $5(23.8)$ & $1(4.8)$ & $6(28.6)$ \\
\hline & $51-60$ & $2(9.5)$ & $2(9.5)$ & $4(19.0)$ \\
\hline & $61-70$ & $3(14.3)$ & $1(4.8)$ & $4(19.0)$ \\
\hline & $71-80$ & $0(0.0)$ & $1(4.8)$ & $1(4.8)$ \\
\hline \multirow[t]{2}{*}{ Systemic diseases } & no & $10(47.6)$ & $3(14.3)$ & $13(61.9)$ \\
\hline & yes & $6(28.6)$ & $2(9.5)$ & $8(38.1)$ \\
\hline \multirow[t]{2}{*}{ Oral habits } & smoking & $0(0.0)$ & $1(4.8)$ & $1(4.8)$ \\
\hline & drinking & $0(0.0)$ & $1(4.8)$ & $1(4.8)$ \\
\hline \multirow[t]{2}{*}{ Duration (months) } & $1-6$ & $10(47.6)$ & $4(19.0)$ & $14(66.7)$ \\
\hline & $7-12$ & $6(28.6)$ & $1(4.8)$ & $7(33.3)$ \\
\hline \multirow[t]{7}{*}{ Site of involvement } & labial mucosa and lips & $7(33.3)$ & $3(14.3)$ & $10(47.6)$ \\
\hline & buccal mucosa & $14(66.7)$ & $3(14.3)$ & $17(81.0)$ \\
\hline & gingiva & $15(71.5)$ & $2(9.5)$ & $17(81.0)$ \\
\hline & palatal mucosa & $5(23.8)$ & $2(9.5)$ & $7(33.3)$ \\
\hline & floor of mouth & $4(19.0)$ & $0(0.0)$ & $4(19.0)$ \\
\hline & tongue & $7(33.3)$ & $0(0.0)$ & $7(33.3)$ \\
\hline & extraoral & 9 (42.9) & $5(23.8)$ & $14(66.7)$ \\
\hline \multirow[t]{2}{*}{ Diagnostic methods } & biopsy & $16(76.2)$ & $5(23.8)$ & $21(100)$ \\
\hline & direct immunofluorescent & $8(38.1)$ & $3(14.3)$ & $11(52.4)$ \\
\hline \multirow[t]{3}{*}{ Steroid treatment } & topical & $6(28.6)$ & $4(19.0)$ & $10(47.6)$ \\
\hline & systemic & $2(9.5)$ & $0(0.0)$ & $2(9.5)$ \\
\hline & combination & 8 (38.1) & $1(4.8)$ & 9 (42.9) \\
\hline
\end{tabular}
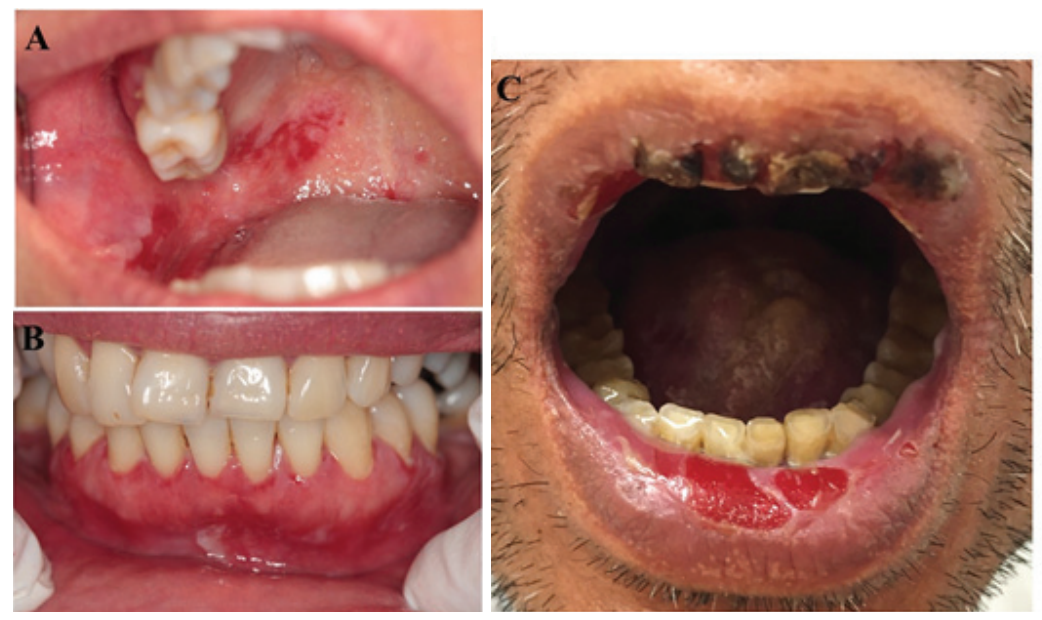

Figure 1. Oral lesions of pemphigus vulgaris on the buccal and palatal mucosa (A), gingiva (B) and lips (C) 

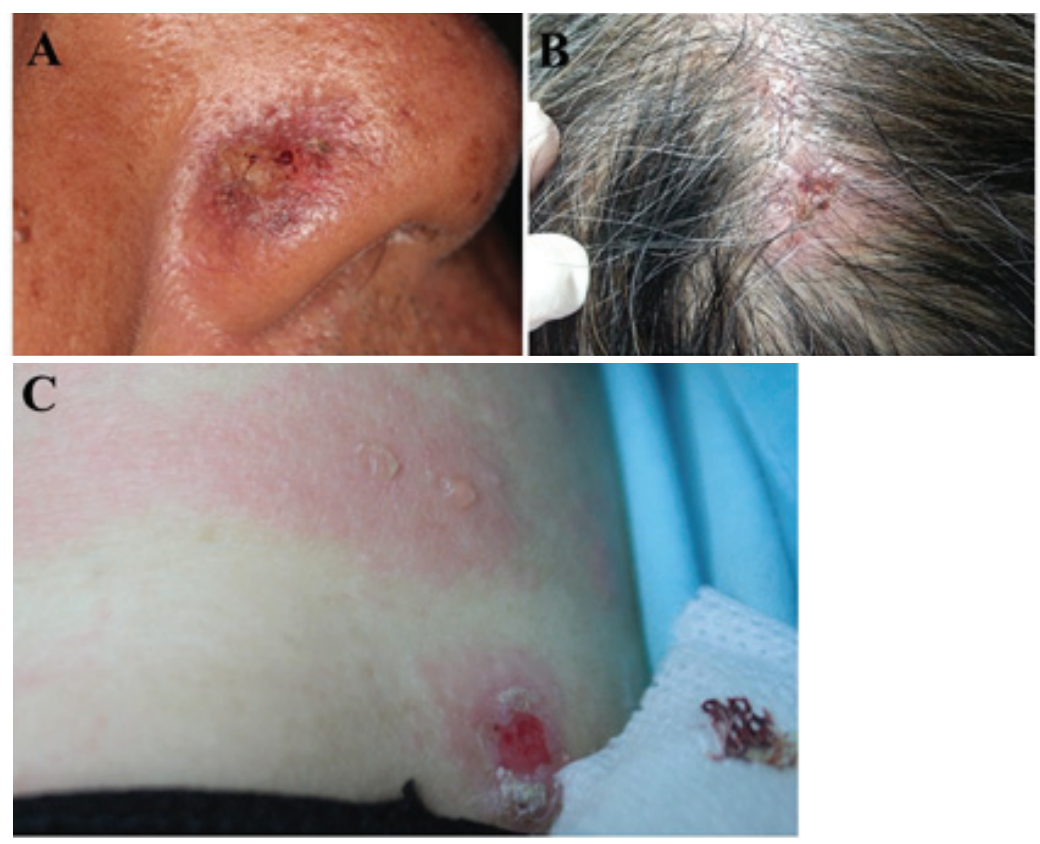

Figure 2. Skin lesions of pemphigus vulgaris on the nose (A), scalp (B) and shoulder (C)

10 patients (47.6\%) were treated with topical steroids, such as $0.1 \%$ fluocinolone acetonide in orabase, $0.1 \%$ triamcinolone acetonide in orabase and dexamethasone mouthwash. In contrast, 2 patients $(9.5 \%)$ were treated with systemic steroids, such as prednisolone and dexamethasone. 9 patients $(42.9 \%)$ were prescribed a combination of topical and systemic steroids. 2 patients (9.5\%) were hospitalized due to the diseases.

\section{Discussion}

Epidemiological and clinical data on oral PV is still limited and varies in different regions of the world. The present study illustrates the demographic and clinical characteristics of oral PV in Thai patients. Pemphigus vulgaris may occur at any age, and disease onset usually occurs between 40 and 60 years of age [1]. This study found that the mean age of PV patients was 47.7 (range 25 to 74 ) years, consistent with Korea's research, which showed a mean age of 47 years [7]. Previous studies from Thailand showed that PV patients' mean age was 37.7 years [8] and 45.4 years [9]. A report from China found that the mean age was 44.2 years [10]. Whereas reports in Turkey, Israel and Poland indicated the mean age was 51.7 years [11], 52.9 years [12] and 54.7 years [13], respectively. However, the study reported that an increased frequency in the elderly and children was observed [1].

Pemphigus vulgaris has been reported more frequently in females, with a difference in the female-male ratio [4]. This study showed that the female-male ratio was $3.2: 1$. The report from Poland showed a female-male ratio of $4.5: 1$ [13]. Previous reports from Thailand showed the female-male ratio was 2:1 [8] and 2.4:1 [9]. Moreover, Turkey and Romania's female-male ratio was 1.2:1 [11] and 1.3:1 [14], respectively.

In several autoimmune diseases, genes encoded on the $X$ chromosome can influence estrogen, which preferentially stimulates Th cells to secrete Th2 cytokines [15]. This sex-specific environmental factor may contribute to the female predominance of autoimmune diseases [16]. A previous study suggested that gender influences disease presentation in PV, supporting genetic and hormonal factors in immune dysregulation and perpetuating the autoimmune phenotype [17].

The progression of PV typically begins with painful oral ulceration. These lesions are persistent, and many patients will develop extraoral or skin lesions over the following weeks or months $[3,6]$. In this study, the most common complaints were oral ulceration, a burning sensation and oral bleeding, of which the range of duration of symptoms was 1 to 12 months (mean: $6 \pm 3.42$ months). Another study showed that the duration of symptoms was 2 days to 15 months (mean: 105 days) [18]. According to the oral involvement, this study found that the buccal mucosa and gingiva were the most common sites of lesions, followed by the lips and labial mucosa, palatal mucosa, tongue and floor of mouth, which was consistent with previous studies $[8,18]$. Nikolsky's sign is a common finding during examination in PV $[1,19]$. However, this sign is not always $100 \%$ reliable for diagnosing PV, but it is suggestive if present [3]. Most of our patients had positive Nikolsky's sign of $90.5 \%$ on the gingival mucosa, only 1 patient had a negative result, and 1 patient did not have a test result.

$66.7 \%$ of our patients had skin lesions on the face, scalp, chest and back for extraoral involvement. Other studies from Thailand reported the extraoral involvement in PV patients was $21.4 \%$ [9] and 22.2\% [8]. Reports from Japan and Turkey found that skin lesions on PV patients amounted to $45.5 \%$ [18] and $76.2 \%$ [11], respectively. The skin involvement of PV might be localized or generalized [3]. Typical distribution of skin lesions is on the upper torso in a shawl-like distribution, heavy on the upper central chest and back in a $V$ shape [6]. The intraepidermal blisters of PV are flaccid, frequently rupturing to leave raw erosions covered with crusting. The face, scalp and pressure points can also be involved $[3,6]$. Other rare extraoral involvements include nail dystrophy, paronychia and subungual hematomas $[1,3]$. Pemphigus is commonly linked to autoantibodies against desmoglein-1 and desmoglein-3. The scalp is a particular location for pemphigus, as hair follicles have localized as hair follicles have localized abundance desmogleins. Scalp involvement was observed in up to $60 \%$ of pemphigus patients, and the lesions may occasionally lead to alopecia $[1,20]$. A previous study suggested that anti-desmoglein-1 autoantibody titers seem to be more useful in showing the extent of the disease and pemphigus activity with mucocutaneous lesions. In contrast, antidesmoglein-3 autoantibody titers can be used for diagnostic purposes [21].

Drugs and various conditions have been reported as trigger factors of pemphigus $[4,5,22]$. Drugs, such as thiol drugs, phenol drugs and others, are the most common cause of pemphigus [5, 22]. Infections, cancer and autoimmune diseases, including Graves's disease and Hashimoto's thyroiditis, myasthenia 
gravis, Sjögren's syndrome and rheumatoid arthritis, may trigger pemphigus initiation in susceptible individuals or be exacerbated in the affected patient $[5,22]$. According to the medical history, $38.1 \%$ of our patients had systemic diseases, including hypertension, diabetes mellitus, gastritis and peptic ulcers, dyslipidemia, ischemic heart disease and hepatitis B. The history of current medications was also used in our patients, comprising amlodipine, losartan, atenolol, hydrochlorothiazide, metformin, acarbose, omeprazole, simvastatin, aspirin, clopidogrel and nitroglycerin. A previous report indicated that PV incidence was increased in individuals with hypertension, diabetes mellitus and dyslipidemia [7]. On the other hand, previous reports suggested that smoking can have a protective effect on patients with pemphigus $[5,22,23]$. In this study, most of the patients were non-smokers and non-drinkers.

The guidelines recommend considering two stages in the management of PV: induction of remission and remission maintenance. In the first stage, corticosteroids are used as treatment. The remission maintenance phase encompasses gradual dose reduction to achieve effective disease control. The goal of $\mathrm{PV}$ treatment is to preserve the remission as long as possible [1, 24]. The first-line treatment of moderate and severe PV cases is systemic corticosteroid therapy $[1,2,4,6]$. For oral lesions, topical steroids may be effective and safe [3]. Most of our patients (61.9\%) were prescribed a combination of topical and systemic steroids, consistent with the recommendation of guidelines and previous studies $[9,11,18,24]$. However, 2 patients $(9.5 \%)$ were hospitalized due to progression of the diseases. Early diagnosis and initiation of treatment are critical for patients and com- pulsory for a promising prognosis. Optimized PV patient care requires a multidisciplinary approach [23]. Even though oral involvement is often the first and the high frequency in PV involvement, diagnostic delays are common for patients with oral PV [25]. Education to improve the knowledge and awareness of healthcare providers is necessary.

This report had some limitations: first, the data from two oral medicine clinics from the lower north and northeast of Thailand were analyzed. Therefore, the population of patients with PV was only somewhat representative, as patients without mucous involvement or with predominant skin involvement were not included. Second, the diagnostic methods were questioned, since direct immunofluorescence was not conducted in some patients, and all of the patients did not have circulating autoantibody results. Lastly, the patient follow-up data was omitted, as some patients did not show up for appointments. Therefore, further studies are required to obtain a clearer understanding of these topics.

\section{Conclusions}

The present study illustrates the demographic and clinical characteristics of oral PV in Thai patients in the lower north and northeast of Thailand. This condition is more frequent in the fifth decade of life and is predominant in females. The buccal mucosa and gingiva were the most affected oral involvement. More than half of the patients had skin lesions, and most patients had been prescribed a combination of topical and systemic steroids.

Source of funding: This work was funded from the authors' own resources.

Conflicts of interest: The authors declare no conflicts of interest.

\section{References}

1. Porro AM, Seque CA, Ferreira MCC, et al. Pemphigus vulgaris. An Bras Dermatol 2019; 94(3): 264-278.

2. Venugopal SS, Murrell DF. Diagnosis and clinical features of pemphigus vulgaris. Immunol Allergy Clin North Am 2012; 32(2): 233-243.

3. Kridin K. Pemphigus group: overview, epidemiology, mortality, and comorbidities. Immunol Res 2018; 66(2): 255-270.

4. Alpsoy E, Akman-Karakas A, Uzun S. Geographic variations in epidemiology of two autoimmune bullous diseases: pemphigus and bullous pemphigoid. Arch Dermatol Res 2015; 307(4): 291-298.

5. Buonavoglia A, Leone P, Dammacco R, et al. Pemphigus and mucous membrane pemphigoid: an update from diagnosis to therapy. Autoimmun Rev 2019; 18(4): 349-358.

6. Melchionda V, Harman KE. Pemphigus vulgaris and pemphigus foliaceus: an overview of the clinical presentation, investigations and management. Clin Exp Dermatol 2019; 44(7): 740-746.

7. Lee YB, Lee JH, Lee SY, et al. Incidence and death rate of pemphigus vulgaris and pemphigus foliaceus in Korea: a nationwide, population-based study (2006-2015). J Dermatol 2018; 45(12): 1396-1402.

8. lamaroon A, Boonyawong $\mathrm{P}$, Klanrit $\mathrm{P}$, et al. Characterization of oral pemphigus vulgaris in Thai patients. J Oral Sci 2006; 48(1): 43-46.

9. Kulthanan K, Chularojanamontri L, Tuchinda P, et al. Clinical features and course of pemphigus in Thai patients. Asian Pac J Allergy Immunol 2011; 29(2): 161-168.

10. Zhu X, Pan J, Yu Z, et al. Epidemiology of pemphigus vulgaris in the Northeast China: a 10-year retrospective study. J Dermatol 2014; 41(1): 70-75.

11. Yavuz IH, Yavuz GO, Bayram I, et al. Pemphigus in the eastern region of Turkey. Post Dermatol Alergol 2019; 36(4): 455-460.

12. Kridin K, Zelber-Sagi S, Bergman R. Pemphigus vulgaris and pemphigus foliaceus: differences in epidemiology and mortality. Acta Derm Venereol 2017; 97(9): 1095-1099.

13. Serwin AB, Koper M, Flisiak I. Incidence of pemphigus vulgaris and pemphigus foliaceus in North-East Poland (Podlaskie Province) - a 15-year (2001-2015) bicentric retrospective study. Int J Dermatol 2018; 57(8): 933-937.

14. Baican A, Chiorean R, Leucuta DC, et al. Prediction of survival for patients with pemphigus vulgaris and pemphigus foliaceus: a retrospective cohort study. Orphanet J Rare Dis 2015; $10: 48$.

15. González DA, Díaz BB, Rodríguez Pérez Mdel C, et al. Sex hormones and autoimmunity. Immunol Lett 2010; 133(1): 6-13.

16. Tiniakou $\mathrm{E}$, Costenbader $\mathrm{KH}$, Kriegel MA. Sex-specific environmental influences on the development of autoimmune diseases. Clin Immunol 2013; 149(2): 182-191.

17. Naseer SY, Gill L, Shah J, et al. Gender-based variability in disease presentation in pemphigus vulgaris. J Drugs Dermatol 2014; 13(10): $1225-1230$.

18. Ohki M, Kikuchi S. Nasal, oral, and pharyngolaryngeal manifestations of pemphigus vulgaris: endoscopic ororhinolaryngologic examination. Ear Nose Throat J 2017; 96(3): 120-127.

19. Santoro FA, Stoopler ET, Werth VP. Pemphigus. Dent Clin North Am 2013; 57(4): 597-610.

20. Sar-Pomian M, Rudnicka L, Olszewska M. The significance of scalp involvement in pemphigus: a literature review. Biomed Res Int 2018; 2018: 6154397.

21. Delavarian Z, Layegh P, Pakfetrat A, et al. Evaluation of desmoglein 1 and 3 autoantibodies in pemphigus vulgaris: correlation with disease severity. J Clin Exp Dent 2020; 12(5): e440-e445.

22. Tavakolpour S. Pemphigus trigger factors: special focus on pemphigus vulgaris and pemphigus foliaceus. Arch Dermatol Res 2018; 310(2): 95-106. 
23. Sullivan TP, Elgart GW, Kirsner RS. Pemphigus and smoking. Int J Dermatol 2002; 41(8): 528-530.

24. Popescu IA, Statescu L, Vata D, et al. Pemphigus vulgaris - approach and management. Exp Ther Med 2019; 18(6): 5056-5060.

25. Daltaban Ö, Özçentik A, Akman Karakaş A, et al. Clinical presentation and diagnostic delay in pemphigus vulgaris: a prospective study from Turkey. J Oral Pathol Med 2020; 49(7): 681-686.

Tables: 1

Figures: 2

References: 25

Received: 04.02.2021

Reviewed: 11.02 .2021

Accepted: 16.09 .2021

Address for correspondence:

Ruchadaporn Kaomongkolgit, MD, PhD, Assoc. Prof.

Department of Oral Diagnosis

Faculty of Dentistry

Naresuan University

Phitsanulok 65000

Thailand

Tel.: +66 5967472

E-mail:ruchadapornk@nu.ac.th 